\title{
SUGGESTED IMPROVEMENTS IN THE METALLURGICAL MICROSCOPE
}

\author{
By Professor H. Le Chatelier (Paris).
}

The writer has for some considerable time been endeavouring to extend the use of the Microscope in Metallurgical Works. No one to-day will contest the services that Metallography renders to Industry, and it is possible that the sphere of usefulness of this method of investigation could be still further extended by improvements in detail.

The object of this brief note is to point out two possible improvements.

In the first case, to obtain good photomicrographs the use of apochromatic objectives is necessary. These are very costly and many workers hesitate to incur the expense of providing them. Would it not be possible to persuade Manufacturers to design Objectives corrected for some single wave-length of the spectrum?-viz., the blue line of the Mercury Vapour Lamp, which is easily separated from the other rays and which moreover has a considerable actinic effect. Such simple objectives in which it would only be necessary to take into account corrections for spherical aberration could be manufactured as a single lens and would thus be comparatively cheap.

The second improvement, which it is desirable to introduce into an objective used for the examination of metals is to give to the radius of curvature of the back surface such a value as to prevent concentration of the light reflected from this surface. In all Metallurgical Microscopes illumination must necessarily be effected through the objective. This is a new condition and consequently one complication more in the construction of objectives, but perhaps it may not be insuperable.

From an entirely opposite point of view it would be very useful if a small handbook were drawn up for the use of those who employ the Microscope, as well as for a few of the Manufacturers, such a manual explaining the essential properties of the instrument. Every day the grossest errors are made in this connection. A great number of experimenters imagine that a Microscope Objective can be used like a thin lens. They forget that every objective is constructed to give an image at a fixed point, this being 16 or 25 centimetres according to the country of manufacture. We frequently see photographs taken with a Microscope objective, in which the adjustment (tube length) is changed so as to project the image a greater or lesser distance according to the magnification it is desired to obtain. Now, on the contrary, the extension of the Microscope should always remain invariable and a projection eye-piece used for taking the photomicrograph. The distance of the two lenses of this eye-piece should be adjusted according to the magnification desired. 
Another practice which should be no less condemned when using the Metallurgical Microscope is that of reflecting the luminous pencil at right angles by means of a total reflection prism placed in the path of the pencil of light, instead of employing a silvered reflecting mirror. The former method completely changes the working of an objective by making the pencil of rays pass through a piece of glass many centimetres thick. The objective is calculated for working in air and not in glass.

These errors are not very important when the examination is simply by the naked eye, because the eye has an extraordinarily high degree of accommodation. This, however, is not the case in photography. Frequently the sharpness of image that ought to be possible where objectives are properly used is far from being obtained.

To sum up : hitherto Microscopes have only been seriously investigated for the examination of transparent objects and it would be highly desirable if this study could be resumed and extended with a view to the examination, by reflection, of polished opaque bodies like metals. 